\title{
Condom catheter: a simple and efficacious alternative of hysterectomy in postpartum haemorrhage
}

\author{
Neeta Natu, Vandna Singh*
}

Department of Obstetrics and Gynecology, SAIMS and PGI, Indore, Madhya Pradesh, India

Received: 15 April 2019

Revised: 8 May 2019

Accepted: 14 May 2019

\author{
*Correspondence: \\ Dr. Vandna Singh, \\ E-mail: vandana.singh297@gmail.com
}

Copyright: (c) the author(s), publisher and licensee Medip Academy. This is an open-access article distributed under the terms of the Creative Commons Attribution Non-Commercial License, which permits unrestricted non-commercial use, distribution, and reproduction in any medium, provided the original work is properly cited.

\begin{abstract}
Background: The aim of this study was to evaluate the effectiveness of the condom catheter in treating postpartum hemorrhage refractory to medical treatment.

Methods: This prospective study included 21 women with postpartum hemorrhage treated with a condom catheter as a conservative therapeutic option.

Results: The condom catheter was successful in controlling hemorrhage in $90.4 \%$ of the women. It was effective in all women with vaginal delivery (11 of 12) and highly effective in women with uterine atony who did not respond to medical uterotonic treatment (6 of 7 women).

Conclusions: Its ease of use and high effectiveness make the condom catheter a useful approach for the conservative management of acute postpartum hemorrhage. This device reduces bleeding, shortens the hospital stay and avoids the need for surgical management.
\end{abstract}

Keywords: Condom catheter, Peripartam hysterectomy, Postpartum hemorrhage, Uterine atony, Uterine tamponade

\section{INTRODUCTION}

Postpartum hemorrhage (PPH), an obstetric emergency that can complicate vaginal or cesarean deliveries, accounts for $25 \%$ of all maternal deaths worldwide. ${ }^{1}$ Initial management of atonic PPH included resuscitative measures, correction of hypovolemia with intravenous fluid/blood transfusion, uterotonic, uterine massage and/or bimanual compression in majority of case, women who do not respond to uterotonic medical treatment, a variety of procedures, such as arterial embolization, surgical ligation of the uterine arteries or obstetric hysterectomy, may be used. Condom catheter a fluidfilled structure exerting a tamponade effect to stop bleeding is used in the management of PPH. With aseptic precautions, a condom was rolled over a Foley's catheter (no. 18) and tied with a cotton thread on two sites $1 \mathrm{~cm}$ apart and then it is inserted into the uterus (Figure 1). The catheter was tightened by an umbilical clamp or cotton string and taped to the thigh. Indwelling catheter was left in bladder until the condom tamponade was in place. Fundal height was marked on abdomen. To keep the balloon in situ, the vaginal cavity was filled with roller gauze and cotton pad. If bleeding continues, this vaginal pack will usually become soaked with blood, and if profuse, it will trickle through the introitus to soak the outside pad and undergarments. ${ }^{2-4}$ Take care not to kink the plastic tubing. Now change position of patient from lithotomy to supine to make more comfortable. The fluid source was kept at $60 \mathrm{~cm}$ above the abdomen with patient 
in lying down position, and now the condom balloon was inflated. 5,6 Airway needle was applied to fluid source to open the system to environmental pressure, Balancing the hydrostatic pressure with uterine tone and balloon inflated with $250-300 \mathrm{ml}$ of isotonic saline until the bleeding was controlled. The condom catheter was then removed after $24-48$ hours. ${ }^{7,8}$ The main advantages for the condom catheter are its easy transvaginal or transabdominal insertion with cesarean section, which can bring about rapid tamponade of the uterine cavity, simplify control of the bleeding and avoid the need for other more invasive procedures, such as hysterectomy. ${ }^{9}$

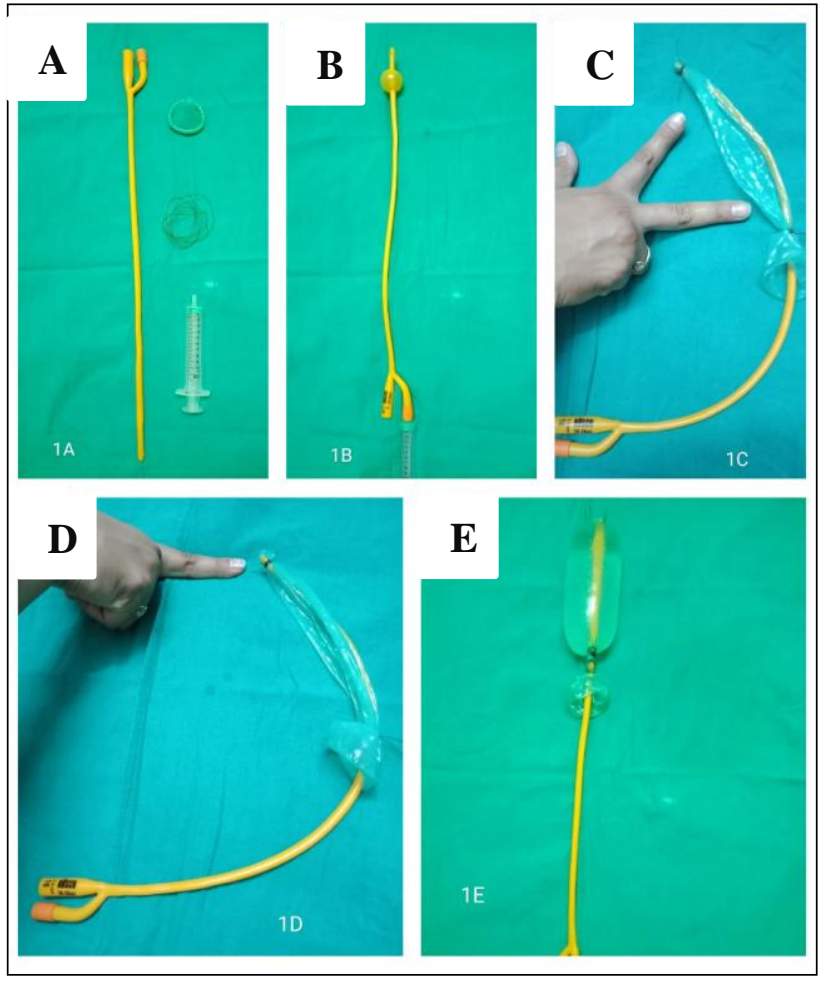

Figure 1: Steps of formation of condom catheter. (A): Equipment's used to prepare condom catheter balloon, (B): Inflation of Foley's catheter balloon followed by rupture to create a new port, (C): Picture shows two tied end with thread, (D): Picture shows draining end, (E): Fully formed condom catheter balloon.

\section{METHODS}

This single-arm prospective study was carried out at the Department of Obstetrics and Gynaecology, SAIMS Medical College and hospital, Indore, Madhya Pradesh, India, from July 2018 to March 2019. The institutional ethics committee approved the study protocol. Total 21 cases were registered for study. Demographic and epidemiological data were recorded in a proforma. Women were considered candidates for treatment with a condom catheter balloon if they had PPH that did not respond to standard management consisting of uterine massage, volume replacement and uterotonic medical treatment. The protocol we followed was intravenous oxytocin or carbetocin, followed by intramuscular methylergometrine, if the bleeding was not controlled. If bleeding still persisted, intramyometrial prostaglandins (carbopost) were used, and if this did not control the bleeding, misoprostol was given rectally.

Study design was prospective. Actual enrolment of 21 participanrts. Model was single group assignment Masking none (open label). Primary purpose was treatment.

\section{Inclusion criteria}

- Women with vaginal delivery

- Women with cesarean section

- Women underwent atonic PPH

\section{Exclusion criteria}

- $\quad$ Patient in shock

- Other than atonic PPH cases

- Patient do not give consent for method

\section{Data management and analysis}

The data were entered in Microsoft excel and further statistical analysis was done using the software SPSS.

\section{RESULTS}

Twenty one cases were identified in which condom catheter balloon was used (Figure 2).

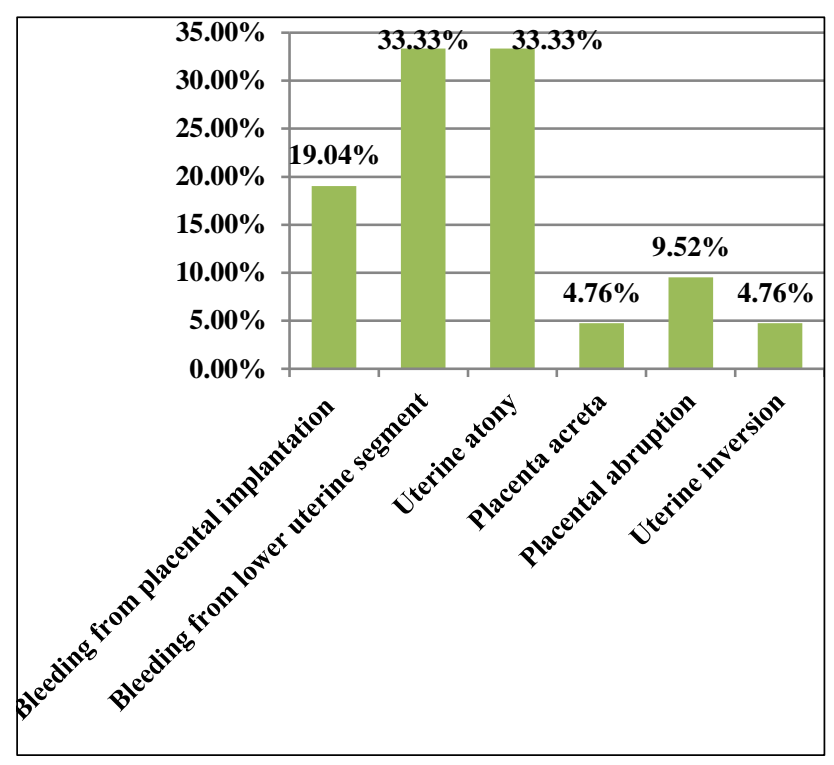

Figure 2: Indication for using condom catheter.

Mean maternal age was $36.5 \pm 6$ years (range $24-38$ years). Of the women for whom the balloon was used, 8 $(38.09 \%)$ were nullipara. Mean gestational age at the time of delivery was 39 weeks (range 36-41 weeks). The main 
risk factors associated with PPH were cesarean delivery (23.08\%), induced labor $(19.05 \%)$, previous cesarean delivery (19.05\%), multiple gestation (14.29\%), multiparous patient $(9.52 \%)$, use of assisted reproduction techniques $(9.52 \%)$ and hypertension $(4.76 \%)$ (Figure 3$)$.

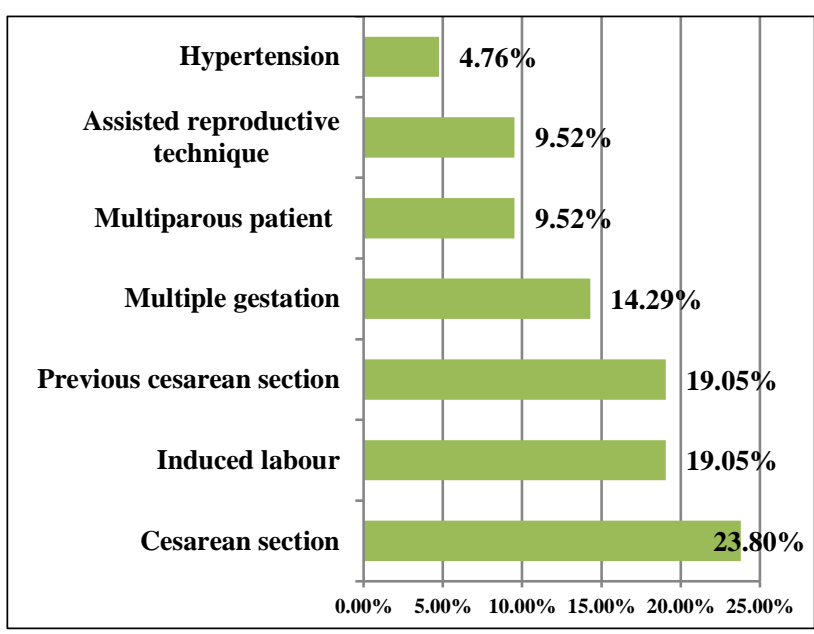

Figure 3: Risk factor for postpartum hemorrhage.

Onset of labor was spontaneous in 8 woman and induced in 4 (with oxytocin in all cases), and cesarean delivery was used for 9 women. The main indication for inducing labor was premature rupture of the membranes in one women, meconium-stained amniotic fluid in one, placental abruption in one and pre-eclampsia in one (Figure 4).

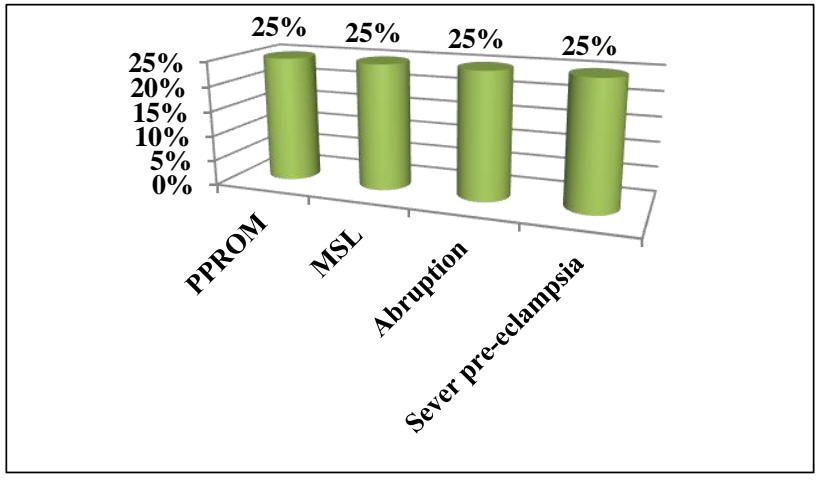

Figure 4: Induction of labour.

Mean duration of induction before the second stage began was $11 \pm 6 \mathrm{~h}$ (range 5-17 h). Of the 21 deliveries, 12 were vaginal, among them, four was spontaneous and the remaining eight required vacuum extraction. In the other 9 women, labor ended in cesarean delivery. The main indications for ending labor with cesarean delivery were previous cesarean section $(44.44 \%)$, failure of induction (22.22\%), Breech presentation (11.11\%), CPD (11.11\%) and placenta previa (11.11\%) (Figure 5). Delivery of the placenta was spontaneous in eight cases (38\%), manual in thirteen cases(62\%) (Figure 6). The most frequent indication for using the condom catheter balloon was bleeding from the lower uterine segment followed by bleeding due to uterine atony and then at the placental implantation site. We also used condom catheter in some specific cases like placental abruption (2 cases), placenta acreta ( 1 case) and one case of uterine inversion.

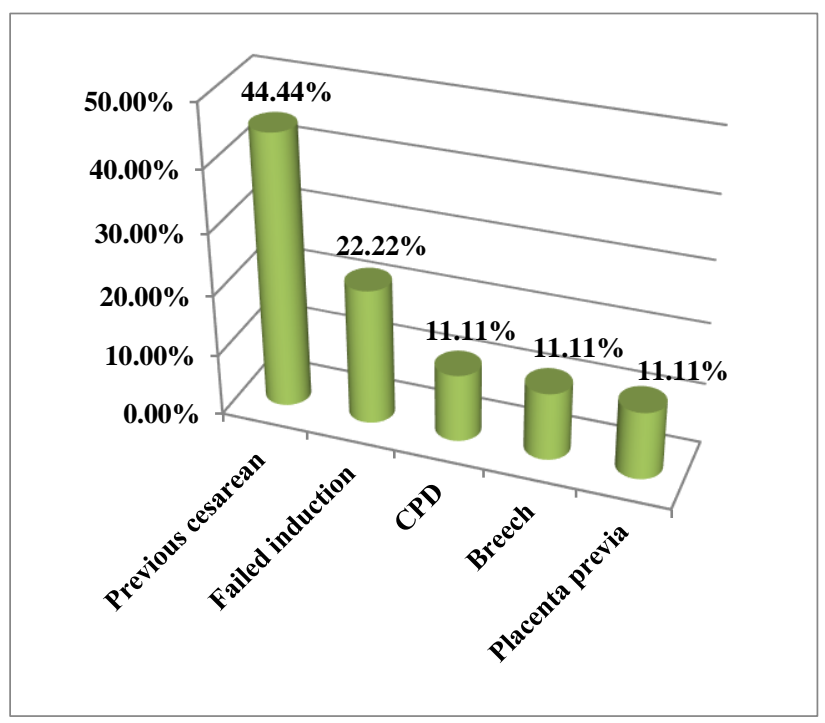

Figure 5: Indication of cesarean section.

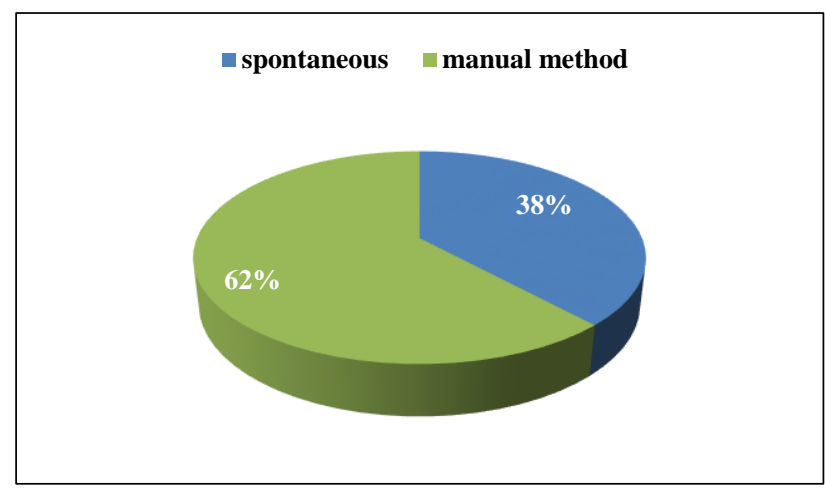

Figure 6: Placenta separation.

The mean volume of saline solution used to fill the balloon initially was $250 \pm 50 \mathrm{~mL}$ (range $150-300 \mathrm{~mL}$ ), and the time during which the balloon remained inflated was $24 \pm 16 \mathrm{~h}$ (range $60 \mathrm{~min}$ to $48 \mathrm{~h}$ ). A mean volume of $150 \pm 50 \mathrm{~mL}$ blood (range $30-300 \mathrm{~mL}$ ) was drained while the balloon and catheter were in place. While the balloon was in place, uterotonic treatment with oxytocin was given by continuous perfusion. According to our definition of effectiveness as control of PPH not requiring any further non-pharmacological intervention, the condom catheter balloon was effective in $85.71 \%$ of the cases (18 of 21 women). Of the three women for whom the balloon failed to control PPH, one was treated with surgical ligation of the uterine arteries and two required Peripartam hysterectomy (Figure 7).

There were no complications related with use of the balloon. Mean hemoglobin concentration for the whole group after delivery was $7.6 \pm 2 \mathrm{~g} / \mathrm{dL}$, and 9 women 
required blood transfusion. Mean length of hospital stay for the whole group was $7 \pm 5$ days (range 4-12 days).

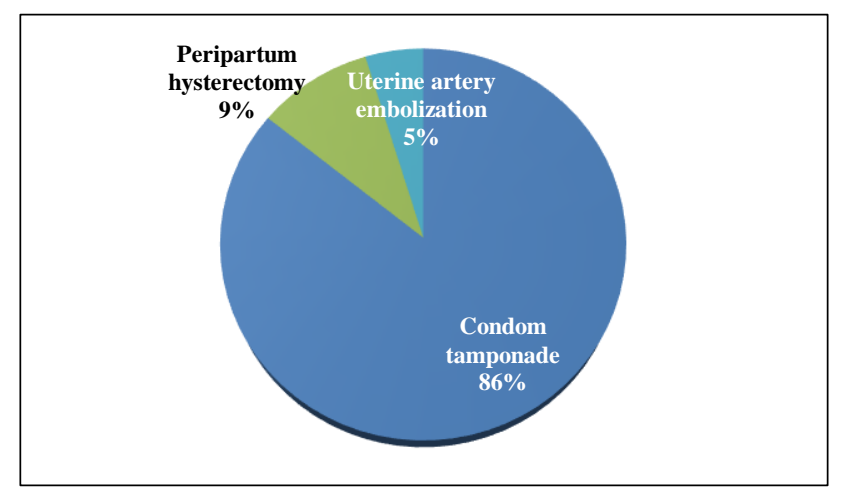

Figure 7: Overall scenario of patients.

\section{DISCUSSION}

PPH a major composite of obstetric hemorrhage- is ubiquitous as it can kill even a healthy woman within 2 $\mathrm{hr}$, if unattended. Although most of the patient treated successfully with conservative measures, such as medications, $10 \%$ of the women with $\mathrm{PPH}$ require major surgical interventions and even hysterectomy to save their lives. ${ }^{10}$ The recommendation is a step-wise approach to management of post-partum hemorrhage from less invasive therapies like uterine massage and uterotonic drugs to more invasive ones like arterial embolization, uterine compression sutures, uterine artery ligation and ultimately hysterectomy. Condom tamponade is an effective intermediate therapy option that can be implemented by frontline health workers with minimal training while surgical procedures are invasive, involve laparotomy and require expertise. The idea of using a condom as a balloon tamponade was first generated and evaluated in Bangladesh in 2001 by Akther et al, in response to the high cost of commercially available UBT (uterine balloon temponade) devices. ${ }^{11,12} \mathrm{We}$ found that balloon tamponade was highly effective in the management of postpartum hemorrhage unresponsive to standard therapy. Further, balloon tamponade was highly successful (6/7 cases) in controlling hemorrhage due to uterine atony and lower uterine segment bleeding (6/7) when the catheter was properly placed. ${ }^{13,14}$

It was also effective as an adjunctive therapy in cases of, placenta accreta, placenta previa, placental implantation site and uterine inversion. ${ }^{15}$ Our study offers practical data on catheter placement including the range of volumes used to inflate the balloons and duration of therapy. In our study, it is observed that timely use of condom tamponade can reduce the maternal morbidity and mortality. The 'golden first hour' is the time at which resuscitation must be commenced to ensure the best chance of survival. The probability of survival decreases sharply if the patient is not effectively resuscitated during the golden first hour of delivery. ${ }^{16}$ Condom tamponade is a quick, minimally invasive, easy procedure and lifesaving if carried out in time; especially in settings where blood transfusion and surgical facilities may not be readily available. Multiple studies have suggested that many deaths and morbidity (organ failure, transfusion complications, thrombosis, ARDS, sepsis, anemia, intensive care, and prolonged hospitalization) associated with PPH could be prevented with prompt recognition and more timely and adequate treatment. ${ }^{17}$

World Health Organization (WHO), the International Federation of Gynecology and Obstetrics (FIGO) and the Royal College of Obstetricians and Gynecologists (RCOG) all recommend a uterine balloon tamponade (UBT) if uterotonic and uterine massage fail to control bleeding. Undoubtedly, UBT is an effective treatment option for control of PPH and it has obtained satisfactory results particularly in the management of uterine atony.

In our study, result is comparable to the following studies: Rathore AM et al, a prospective study in a tertiary hospital in India on 18 patients found that condom tamponade was $94 \%$ successful in controlling hemorrhage. ${ }^{18}$ Shaguftha Yasmeen Rather et al, evaluated the success of condom tamponade in arresting intractable PPH on 26 cases and concluded that condom tamponade is simple, cheap and effective (success rate of 96.2\%) alternative to manage a dreaded obstetric complication like PPH encountered frequently and to reduce maternal mortality and morbidity associated with $\mathrm{PPH}$ effectively. ${ }^{19}$ Shivker et al, , conducted a study at tertiary care facility in India to evaluate the efficacy of condom tamponade on 73 patients with intractable $\mathrm{PPH}$; concluded condom tamponade was successful in controlling PPH in 68/73 patients $(93.2 \%) .{ }^{20}$

The advantages of using balloon tamponade include its ease of use, rapid placement, immediate results, and ability to measure further bleeding after the catheter is placed. If bleeding persists, laparotomy, uterine artery embolization and Peripartam hysterectomy should be considered. In our study condom tamponade effectively controlled bleeding (unresponsive to uterotonics) in $85.71 \%$ cases.

Only 3 out of 21 cases had continued bleeding despite use of condom tamponade. First case bleeding continued from lower uterine segment despite condom tamponade administration (even after 1 hour of applying tamponade) and hence laparotomy and surgical intervention was done (Bilateral Uterine Artery ligation done); second case was a referred case after delivery she had atonic PPH managed with uterotonics and was not responding hence was referred to our hospital; condom temponade was administered to her and she was in shock and bleeding was persisting despite condom application; after stabilizing general condition she undergone surgical intervention to stop the ongoing bleeding; peripartam Hysterectomy was performed and in third case bleeding from placental implantation site despite of 30 min apply of balloon temponade with uterotonic bleeding not 
controlled patient multiparous her family completed so decision of Peripartam hysterectomy was taken. In the case report of Bakri et al, the indications for the use of the catheter were limited to postpartum hemorrhage in patients with a low-lying placenta or previa in the absence of uterine atony. ${ }^{21}$ Condous studied 16 cases of postpartum hemorrhage and used balloon tamponade not only for uterine atony but also for other conditions. ${ }^{22}$ Seror et al, reported on 17 patients with atony or retained products treated with balloon tamponade for postpartum bleeding who failed medical therapy ${ }^{23}$.We did not include a comparison group of patients with similar blood loss who did not have balloon temponade. This was the main limitation of our study. Future studies should address the duration of therapy and the amount of fluid or balloon pressure required to stop hemorrhage.

\section{CONCLUSION}

Because of the ease of use, low cost, availability, low morbidity and success of these catheters, we recommend that labor and delivery units stock balloon catheters for use in cases of postpartum hemorrhage unresponsive to medical treatment.

Funding: No funding sources

Conflict of interest: None declared

Ethical approval: The study was approved by the Institutional Ethics Committee

\section{REFERENCES}

1. World Health Organization (WHO). Attending to 136 million births, every year. Chapter 4. In: Make Every Mother and Child Count: TheWorld Report 2005, Geneva, Switzerland: WHO, 2005; 62-63.

2. Johanson R, Kumar M, Obhrai M, Young P. Management of massive postpartum haemorrhage: use of a hydrostatic balloon catheter to avoid laparotomy. BJOG. 2001;108(4):420-2.

3. Airede LR, Nnadi DC. The use of the condomcatheter for the treatment of postpartum haemorrhage: the Sokoto experience. Trop Doct. 2008;38(2):84-6.

4. Bagga R, Jain V, Sharma S, Suri V. Postpartum haemorrhage in two women with impaired coagulation successfully managed with condom catheter tamponade [2]. Indian J Med Sci 2007;61(3):157-60.

5. Turner GD. Uterine haemorrhage controlled by an intrauterine balloon insufflated with hot water. Hosp Med. 2002;63(7):438.

6. Akhter S, Begum MR, Kabir Z, Rashid M, Laila TR, Zabeen F. Use of a condom to control massive postpartum haemorrhage. Med Gen Med. 2003;5(3):38.

7. Belfort MA, Dildy GA. Postpartum hemorrhage and other problems of the third stage. In: James DK, Steer PJ, Weiner CP, eds. High risk Pregnancy:
Management options. 4th edition. Saunders Elsevier; 2011:1283-1312.

8. Nahar N, Yusuf N, Ashraf F. Role of intrauterine balloon catheter in controlling massive $\mathrm{PPH}$ : experience in Rajshahi Medical College Hospital. The Orion Med J. 2009;32(3):682-3.

9. Sheikh L, Najmi N, Khalid U, Saleem T. Evaluation of compliance and outcomes of a management protocol for massive postpartum haemorrhage at a tertiary care hospital in Pakistan. BMC Pregnancy Childbirth. 2011;11:28.

10. Sequeira E, Patel Y. Acute puerperal uterine inversion: a case report. East Afr Med J. 2007;84:194-6.

11. Hostetler DR, Bosworth MF. Uterine inversion: a life threatening obstetric emeregnecy. J Am Board Fam Pract. 2000;13(2):120-3.

12. Rossen J, Okland I, Nilsen OB, Eggebø TM. Is there an increase of postpartum hemorrhage, and is severe hemorrhage associated with more frequent use of obstetric interventions? Acta Obstet Gynecol Scand. 2010;89(10):1248-55.

13. Georgiou C. Balloon tamponade in the management of postpartum haemorrhage: a review. BJOG. 2009;116(6):748-57.

14. Doumouchtsis SK, Papageorghiou AT, Vernier C, Arulkumaran S. Management of postpartum hemorrhage by uterine balloon tamponade: prospective evaluation of effectiveness. Acta Obstet Gynecol Scand. 2008;87(8): 849-55.

15. Dabelea V, Schultze PM, McDuffie RS. Intrauterine balloon tamponade in the management of postpartum hemorrhage. Am J Perinatol. 2007;24(6):359-64.

16. Rachagan SP, Sivanesaratnam V, Kok KP, Raman S. Acute puerperal inversion of the uterus. An obstetric emergency. Aust NZJ Obstet Gynaecol. 1988;28:2932.

17. Yoong W, Ridout A, Memtsa M, Stavroulis A, ArefAdib M, Ramsay-Marcelle Z, et al. Application of uterine compression suture in association with intrauterine balloon tamponade ('uterine sandwich') for postpartum hemorrhage. Acta Obstet Gynecol Scand. 2012;91(1):147-51.

18. Rathore AM, Gupta S, Manaktala U, Gupta S, Dubey C, Khan M. Uterine tamponade using condom catheter balloon in the management of non-traumatic postpartum hemorrhage. J Obstet Gynaecol Res. 2012 Sep;38(9):1162-7.

19. Rather SY, Qadir A, Parveen S, Jabeen F. Use of condom to control intractable PPH. JK Science 2010;12(3):127-9.

20. Shivkar KS, Khadilkar SS, Gandhewar M. Pressure balloon therapy in uncontrolled obstetrical haemorrhage. J Obstet Gynecol India 2003;53:33841.

21. Bakri YN, Amri A, Abdul Jabbar F. Tamponade balloon for obstetrical bleeding. Int J Gynaecol Obstet. 2001;74(2):139-42.

22. Condous GS, Arulkumaran S, Symonds I, Chapman $\mathrm{R}$, Sinha A, Razvi K. The "temponade test" in the 
management of massive postpartum hemorrhage. Obstet Gynecol. 2003;101(4):767-72.

23. Seror J, Allouche C, Elhaik S. Use of SengstakenBlakemore tube in massive postpartum hemorrhage: a series of 17 cases. Acta Obstet Gynecol Scand 2005;84(7):660-4.
Cite this article as: Natu N, Singh V. Condom catheter: a simple and efficacious alternative of hysterectomy in postpartum haemorrhage. Int J Reprod Contracept Obstet Gynecol 2019;8:2493-8. 